\title{
Grain Boundary Issues in Porous Silicon Nitride Ceramics
}

\author{
M. E. Brito
}

National Institute of Advanced Industrial Science and Technology (AIST), Synergy Materials Research Center, Moriyama-ku, Nagoya 463-8687 JAPAN

Detailed ad hoc studies of porous ceramics by transmission electron microscopy (TEM) are hardly found in the available literature. An important type of porous ceramics is that in which the ceramics have undergone an incomplete sintering process. An understanding of boundary formation and its structure will become a crucial step in helping to draw new processing strategies. Different mechanism and kinetics of boundary formation might be found for systems with a significant higher level of impurities or systems where formation of a transient liquid phase is a necessary condition for densification. Here, a study by TEM and related analytical techniques is presented on the microstructure of liquid phase sintered porous silicon nitride.

The detailed method to produce porous $\mathrm{Si}_{3} \mathrm{~N}_{4}$ is given elsewhere [6]. In brief, slurry formed by commercially available $\beta-\mathrm{Si}_{3} \mathrm{~N}_{4}$ whiskers (UBE Industries, Ltd., Tokyo, Japan) mixed with sintering additives (5 wt. $\% \mathrm{Y}_{2} \mathrm{O}_{3}$ and $2 \mathrm{wt} . \% \mathrm{Al}_{2} \mathrm{O}_{3}$ ) is tape cast. The green sheets were stacked and pressed followed for sintering at $1850{ }^{\circ} \mathrm{C}$ under a nitrogen pressure of $1 \mathrm{MPa}$. The resulting microstructure is anisotropic with whiskers well aligned along the casting direction. For the sake of comparison, dense $\mathrm{Si}_{3} \mathrm{~N}_{4}$ was produced by the conventional method with commercially available $\alpha-\mathrm{Si}_{3} \mathrm{~N}_{4}$ powder (E-10, UBE Industries, Ltd., Tokyo, Japan). Relative density for porous and dense $\mathrm{Si}_{3} \mathrm{~N}_{4}$ was 86 and $98 \%$, respectively.

Porous specimens for TEM observations were prepared by ion beam milling technique. The specimens were examined using a JEM-2010F (Jeol Ltd., Tokyo, Japan) Field Emission-STEM operated at $200 \mathrm{kV}$. The microscope was equipped with a NORAN ultra-thin-window (UTW) type EDS detector controlled by a NORAN Vantage system for signal processing. Elemental maps were obtained by scanning the focused probe $(0.7 \mathrm{~nm})$ in a raster fashion across the specimen. High angle annular dark-field (HAADF) images were obtained. The contrast of HAADF images is strongly dependent on the average atomic number of the scatterer encountered by the incident probe.

Figure 1 shows HAADF images of porous, with a coarse microstructure, and dense silicon nitride. The distribution of amorphous phase (identified by electron diffraction techniques) and appearing in the micrograph with the lighter contrast, is rather irregular in the porous material. Presence of the amorphous phase acting as non-permeable seal in the pores edge (covering the pore walls in 3-D), as revealed by EDS elemental maps of Fig.2, explain difficulties in obtaining open porosity in these materials. The author has reported on the presence of "special boundaries" free of the ubiquitous glassy phase in highly anisotropic silicon nitride, where adjacent grains present a similar orientation [2]. In spite of the higher degree of grain alignment, the EDS analysis reveals an unusual thick film (2.5-6 nm in thickness) in between adjacent grains, which is indication of a more relaxed microstructure due to porosity, or a higher oxygen activity in zones adjacent to pores. Figure 3 depicts EDS elemental map of Y the intergranular amorphous film (IGF). The IGF's thickness is evaluated 
directly from the picture taken under boundary edge-on condition. The presence of a thick IGF would affect negatively the mechanical properties of porous silicon nitride at elevated temperatures. [3]

\section{References}

[1] Y. Shigegaki et al., J. Am. Ceram. Soc. 80 [2] (1997)495.

[2] M E Brito et al., Microsc. Microanal. 6 (Suppl. 2) (2002) 420.

[3] I am greatly indebted to Dr. Y. Shigegaki of IHI Ltd., Japan for supplying all the samples. This work has been supported by AIST, METI, Japan, as part of the Synergy Ceramics Project. The author is a member of the joint Research Consortium of Synergy Ceramics.

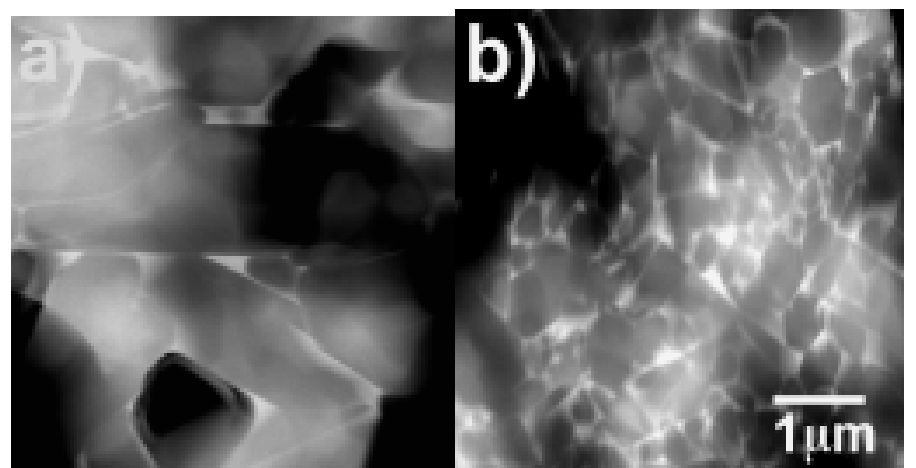

Figure 1 HAADF images of a) porous and b) dense silicon nitride

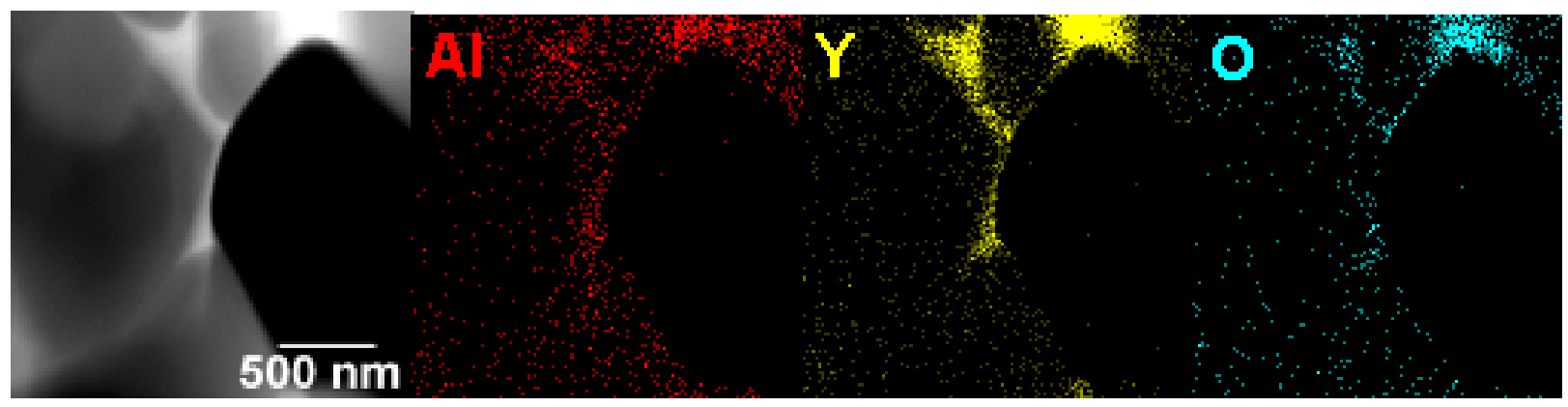

Fig. 2 EDS elemental maps in the vicinity of a pore in the porous silicon nitride.

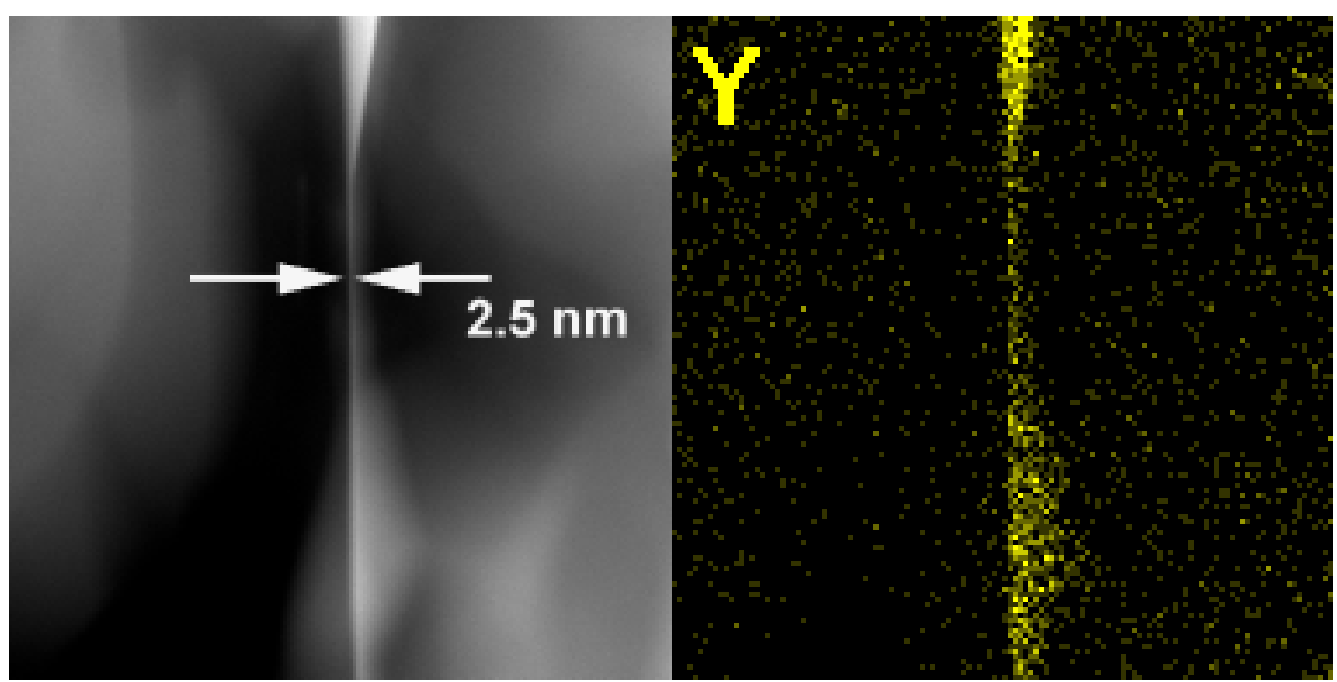

Fig. 3 Yttrium EDS map for the intergranular amorphous phase. 